\title{
Modified PSO Algorithm for Real-time Energy Management in Grid-connected Microgrids
}

\author{
Md Alamgir Hossain ${ }^{1,2, *}$, Hemanshu Roy Pota $^{1}$, Stefano Squartini ${ }^{3}$ and Ahmed Fathi Abdou ${ }^{1}$ \\ ${ }^{1}$ School of Engineering $\&$ Information Technology, The University of New South Wales, Canberra, ACT 2610, Australia \\ ${ }^{2}$ Department of Electrical $\&$ Electronic Engineering, Dhaka University of Engineering and Technology, Gazipur, Bangladesh \\ ${ }^{3}$ Department of Information Engineering, Università Politecnica delle Marche, Ancona, Italy
}

\begin{abstract}
Real-time energy management of a converter-based microgrid is difficult to determine optimal operating points of a storage system in order to save costs and minimise energy waste. This complexity arises due to time-varying electricity prices, stochastic energy sources and power demand. Many countries have imposed real-time electricity pricing to efficiently control demand side management. This paper presents a particle swarm optimisation (PSO) for the application of real-time energy management to find optimal battery controls of a community microgrid. The modification of the PSO consists in altering the cost function to better model the battery charging/discharging operations. As optimal control is performed by formulating a cost function, it is suitably analysed and then a dynamic penalty function in order to obtain the best cost function is proposed. Several case studies with different scenarios are conducted to determine the effectiveness of the proposed cost function. The proposed cost function can reduce operational cost by $12 \%$ as compared to the original cost function over a time horizon of 96 hours. Simulation results reveal the suitability of applying the regularised PSO algorithm with the proposed cost function, which can be adjusted according to the need of the community, for real-time energy management.
\end{abstract}

Keywords: Converter-based microgrids, renewable energy sources, optimum battery control, real-time energy management, and particle swarm optimisation.

\section{Abbreviations}

The following abbreviations are used in this manuscript:

PSO

Particle swarm optimisation

RESs

Renewable energy sources

ESS Energy storage system

SOC State of charge

ADP Adaptive dynamic programming

I-DEMS Intelligent dynamic energy management system

PV Photovoltaic

PCC Point of common-coupling
EMS Energy management system

$B L_{o} \quad$ Initial battery energy level

$B L_{\max } \quad$ Maximum battery energy level

$B L_{\min } \quad$ Minimum battery energy level

$P_{w T} \quad$ Total wind power

$P_{s T} \quad$ Total solar power

$P_{g} \quad$ Import/export grid power

$L(t) \quad$ Load at time $t$

\footnotetext{
${ }^{*}$ Corresponding author

Email address: Md.Hossain6@student.adfa.edu.au or alamgir_duet@hotmail.com (Md Alamgir Hossain ${ }^{1,2, *}$ )
} 


$\begin{array}{llll}\text { Cost } & \text { Objective function } & P_{c, \text { max }} & \text { Maximum charging rate } \\ u & \text { Battery command signals } & P_{d, \max } & \text { Maximum discharging rate } \\ f_{p c}(C) & \text { Penalty cost function } & \eta & \text { Efficiency } \\ \text { RRTP } & \text { Real-time residential electricity price } & C F & \text { Constriction factor } \\ \text { WT } & \text { Wind turbine } & \phi & \text { Constriction co-efficient } \\ \text { SI } & \text { Solar irradiation } & & \end{array}$

\section{Introduction}

The penetration of intermittent renewable energy sources (RESs), such as wind and solar, into existing distribution networks has increased significantly to meet the growing electricity demand and fulfil the target of reducing greenhouse gas emissions 1, 2. However, these RESs, depending on weather conditions and time of day, are stochastic in nature, resulting in variable power generation. Random application of RESs with this intermittent power generation can create difficulty in control, operation and management of the distribution network [3. To minimise complexities on an existing power system, the concept of a microgrid has been introduced [4]. A microgrid (consisting of small-scale emerging generators, loads, energy storage elements and a control unit) is a controlled, small-scale power system that can be operated in an islanded and/or grid-connected mode in a defined area to facilitate the provision of supplementary power and/or maintain a standard service [5]. In the case of grid-tied microgrids, power can be exchanged with the main grid depending on electricity prices. Although electricity price per $\mathrm{kWh}$ is generally fixed, it may vary over the time to have better demand management with the help of smart meter technologies capable of measuring power generation and demand in each time instant [6.

The central control unit in a microgrid is responsible for efficient power management with the help of an energy storage system (ESS) during the operation of the grid-following or grid-forming mode [7. The application of ESSs increases the stability of the grid utility, upgrades the capacity of transmission lines, allows RES penetration, levels load curves, mitigates voltage fluctuation, and improves power quality and reliability 8. An ESS absorbs surplus power and then dispatches it later when input sources are not available, e.g., at night time for solar PV panels. In addition, energy can be stored when electricity prices are low and sold during the hours of high prices to minimise operational costs, allowing more flexible and reliable energy management for a community microgrid. To control the battery energy optimally for reducing electricity costs of investors, the formulation of the cost function considering charging and discharging factors needs to be carefully analysed in addition to the application of efficient algorithms.

An intelligent energy management for handling uncertainties in microgrids becomes essential to maintain a reliable power supply. Energy management for a microgrid, consisting of solar, wind and battery, involves determining the most economical control of stored energy that reduces operating costs subject to the satisfaction of a number of operating constraints. Although conventional if-else rule-based policies, where initial sets of rules are fixed for different scenarios, are employed, they can not provide an optimal control policy [9, 10]. Generally, in this method, energy is dispatched based on available power and state of charge (SOC) of the battery, and stored if power generation is higher than power demand [11. In addition, abrupt charging and discharging decisions can decrease the lifetime of the storage system without providing maximum benefits. 
To improve optimal control approaches for battery energy, a lot of optimisation algorithms, such as linear 40 programming [12, 13, 14, dynamic programming [15], fuzzy logic [16] and adaptive dynamic control [17, are presented in literature. In [18, an expert-based control approach incorporating operating constraints, such as SOC limits and charging/discharging current limits, is presented to optimally use the storage systems for dispatching RESs smoothly. In [16, a fuzzy logic expert system to minimise the operations cost and emission levels of a microgrid is developed. A power management mechanism using dynamic programming for photovoltaic (PV) systems with storage to allow massive penetration of PV power into a distribution network is described in [19]. In [20, a firefly algorithm to find the optimal operation of a microgrid considering lifetime characteristics of a battery is applied. A batch reinforcement learning algorithm to optimally schedule the battery energy for maximising the self-consumption of the local power generation in a microgrid is presented in 21. The adaptive dynamic programming (ADP) for the residential power management and control that focuses on a home battery management is applied in [22]. ADP is employed in an office building to control battery energy management optimally [23]. An intelligent dynamic energy management system (I-DEMS) is developed to optimally use RESs and an energy storage system for maintaining continuous electricity supply to critical loads 9 .

In most of the literature reported above, the contribution remains in the application of new algorithms with different objective functions and reduction of simulation time to minimise operational costs. Although a formulation of an objective function has a direct involvement in altering results, little attention has been paid when applying it to efficiently control the battery energy. Consequently, slight improvements in their results can be observed when their methods are compared to that of others. To find improved solutions quickly and continuously, this paper proposes a particle swarm optimisation (PSO) that runs any time a new sample is available (in our simulations this occurs with a sampling time equal to $1 \mathrm{~h}$ ) for analysing cost functions and then a penalty function related to a dynamic electricity price is multiplied to the charging component. This is done for optimally charging and discharging the battery energy in order to exchange energy with the grid utility depending on electricity prices and availability of local power generated. The major contributions are listed as follows:

- A regularised PSO algorithm in which a different procedure from the conventional one is presented for the application of real-time energy management for a community microgrid;

- Cost functions, used to determine the output of an optimisation algorithm, for determining charging/discharging energy amount of a battery are analysed; and

- A dynamic penalty function, which can be tuned, depending on energy generation from RESs, to efficiently manage battery energy is proposed to the cost function.

The rest of the paper is organised as follows. Section 2 introduces an overview of a community microgrid. In Section 3, the different components of the microgrid to facilitate the analysis are modelled. Section 4 proposes a cost function with constraints after analysing other cost functions. In Section5 the metaheuristic optimisation algorithm in the standard and regularised forms is presented. Simulation results for the different cost functions in two scenarios are performed in Section 6. Section 7 concludes the study. 


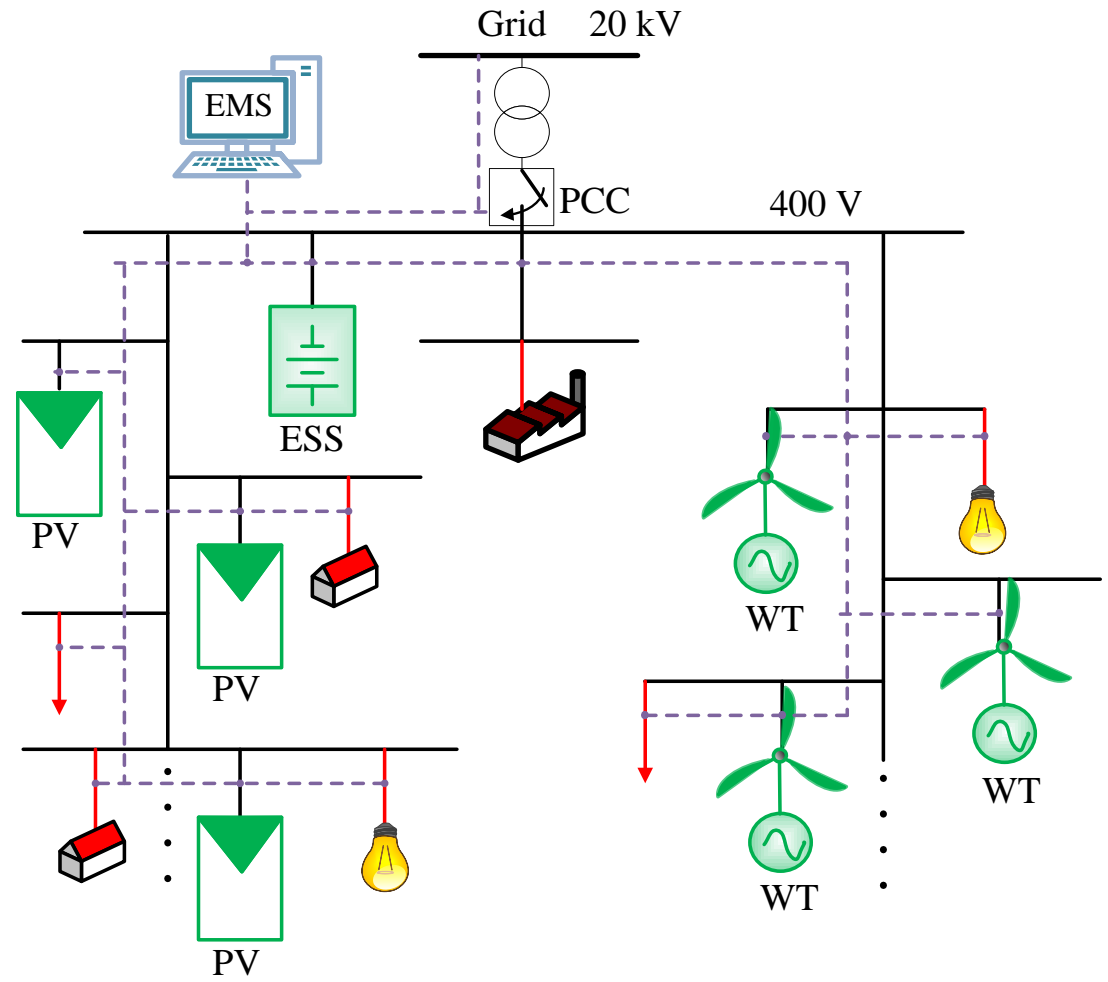

Figure 1: Typical converter-based microgrid.

\section{Description of a community microgrid}

A community microgrid using local resources to meet power demand is illustrated in Figure 1. The community, consisting of twenty houses, has five solar generators $(4 \mathrm{~kW})$ and six wind generators $(5 \mathrm{~kW})$ installed. Thus, the microgrid consists of PV generators, wind generators, an energy storage system, local loads, a main electrical grid and an energy management system (EMS). It is connected to the main grid through a transformer and the point of common coupling (PCC). Although the microgrid is capable of operating either islanded or grid-tied modes, only the latter is taken into account for this paper to exchange powers. The main grid and RESs can supply the power to loads and/or charge the battery. The excess energy after fulfilling local demands from RESs can be sold to the grid in order to reduce operational costs. Due to security and reliability issues of continuous power supply to local loads, in this study, the battery energy is not sold.

The EMS manages energy to ensure the requirement of the local power demand over the time periods and to exchange power with the main grid during the excess and scarcity of the local power generation. Although a number of countries may not approve an energy business with the grid utility due to their rules and regulations, this study considers case studies where there are no obligations for this business rather than an encouragement for microgrid investors. The total capacity for PV generators, wind generators and battery are taken as $20 \mathrm{~kW}$, $30 \mathrm{~kW}$ and $35 \mathrm{kWh}$, respectively. The battery capacity, considered to supply for one hour local load, increases complexities of the analysis due to a small storage system with stochastic power generation. Generally, battery capacity is estimated three times greater than the maximum load demand to maintain continuous supply at least three hours after the loss of grid connection [24. The optimal size determination of the microgrid parameters is beyond the scope of this paper. The parameters of the solar generator, wind generator and battery are given 
Table 1: Input parameters.

\begin{tabular}{|c|c|c|}
\hline Parameter & Value & Unit \\
\hline \multicolumn{3}{|l|}{ PV generators } \\
\hline Total covered area, A & 125 & $\mathrm{~m}^{2}$ \\
\hline Efficiency, $\eta_{s}$ & 16 & $\%$ \\
\hline Maximum power & 20 & $\mathrm{~kW}$ \\
\hline \multicolumn{3}{|l|}{ Wind generators } \\
\hline Cut-in velocity & 3 & $\mathrm{~m} / \mathrm{s}$ \\
\hline Cut-out velocity & 25 & $\mathrm{~m} / \mathrm{s}$ \\
\hline Rated speed & 10 & $\mathrm{~m} / \mathrm{s}$ \\
\hline Maximum power & 30 & $\mathrm{~kW}$ \\
\hline \multicolumn{3}{|l|}{ Battery } \\
\hline Initial energy level, $B L_{o}$ & 17.5 & $\mathrm{kWh}$ \\
\hline Maximum energy level, $B L_{\max }$ & 35 & $\mathrm{kWh}$ \\
\hline Minimum energy level, $B L_{\min }$ & 7 & $\mathrm{kWh}$ \\
\hline Energy capacity & 35 & $\mathrm{kWh}$ \\
\hline Maximum charging rate & 3.5 & $\mathrm{kWh}$ \\
\hline Maximum discharging rate & -3.5 & $\mathrm{kWh}$ \\
\hline
\end{tabular}

in Table 1 .

Although generated power, which is unpredictable and can not directly be handled, may be partially controlled using power curtailment strategy, this study considers operating all RESs in maximum power tracking modes to extract maximum power. As a result, controlling battery energy, which is a crucial issue for the consideration of dynamic electricity prices, available energy and satisfaction of power demand, is the only option for these types of microgrids. The battery can only be operated as one of the following modes at a time:

- Charge modes: the battery can be charged from the grid and/or RESs with an energy quantity which is not beyond the charging rate;

- Discharge modes: the battery supplies energy to loads when prices are high with an energy quantity within the battery discharging rate;

- Inactive modes: there is no activity of the battery energy in this mode as the grid utility directly supplies the electricity to loads at certain hours in order to consider economical perspectives. 


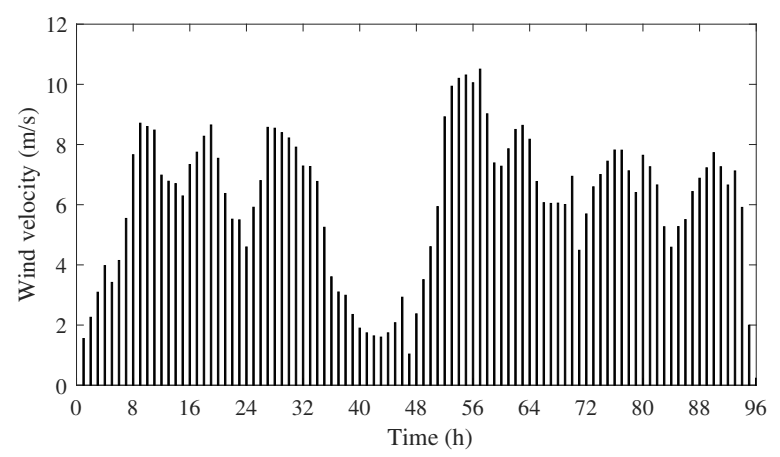

Figure 2: Wind velocity, $v_{o}$.

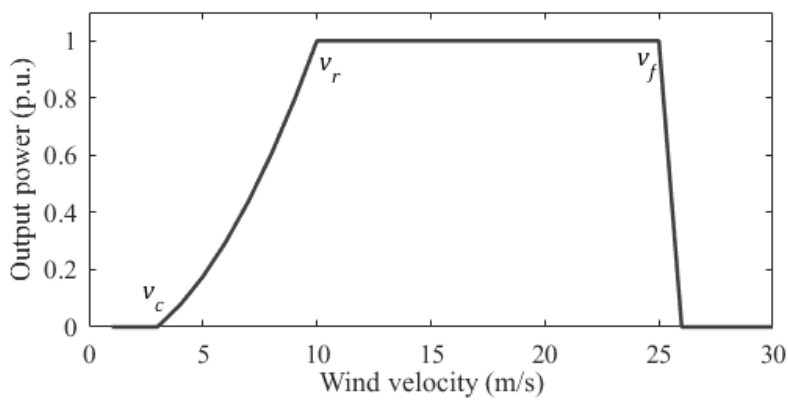

Figure 3: Power curve of wind generator.

\section{System modeling}

\subsection{Wind generators}

Wind power, a free available energy source, is the electric power generated by rotating turbine blades, mounted on a tower at a considerable height, while wind hits the blades of the wind turbine. As wind is stochastic in nature, wind turbines have no control over its generated power. Therefore, power generation by wind turbines solely depends on the availability of wind speed and this varies with heights. The wind speed $v_{1}$ is shown in Figure 2 The wind speed measured at anemometer height needs to be converted to hub heights by the following power law equation [25]:

$$
\frac{v}{v_{o}}=\left(\frac{h}{h_{o}}\right)^{\alpha}
$$
which is a function of parameters 26,27 . The value of $\alpha$ is generally considered as $1 / 7$ for an open space.

Power generated by wind turbines is a function of wind velocity, and a piece-wise function relates the relationship between the wind speed and output power as follows [28:

$$
P_{w}= \begin{cases}0 & \text { if } v_{f} \leq v \text { or } v \leq v_{c} \\ P_{r} * \frac{v^{3}-v_{c}^{3}}{v_{r}^{3}-v_{c}^{3}} & \text { if } v_{c} \leq v \leq v_{r} \\ P_{r} & \text { if } v_{r} \leq v \leq v_{f}\end{cases}
$$

where $P_{r}$ and $v_{r}$ are the rated electrical power and rated wind speed, respectively, and $v, v_{c}$ and $v_{f}$ represent wind speed, cut-in wind speed and cut-off wind speed, respectively. The power curve of a wind generator is illustrated in Figure 3 in which power generation begins at the $v_{c}$ and stops at the $v_{f}$. The output power 


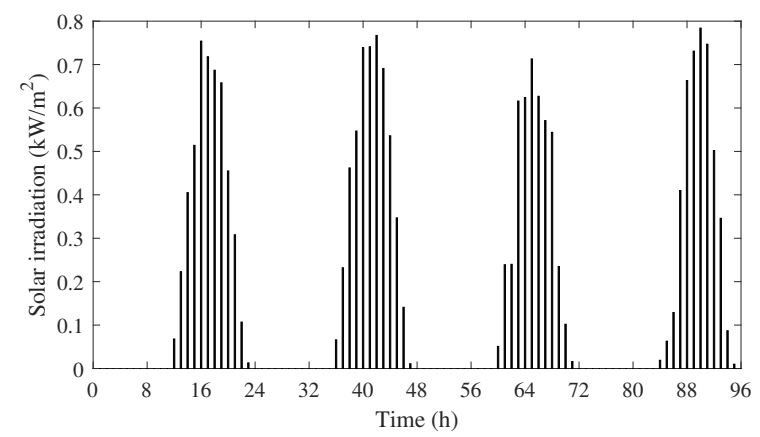

(a)

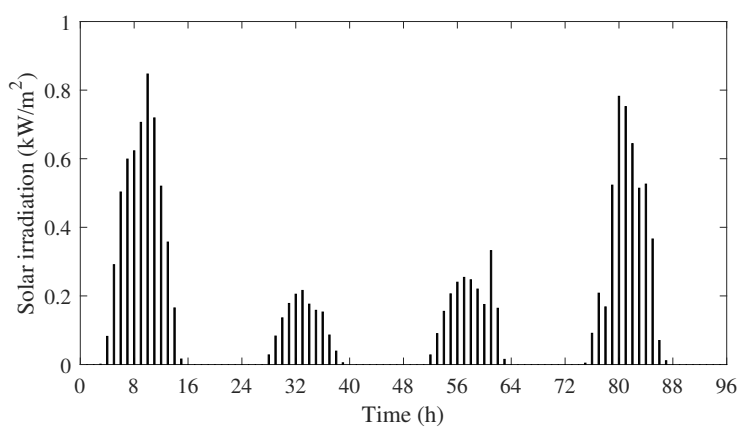

(b)

Figure 4: Solar irradiation over a 96 h horizon (a) Scenario 1 and (b) Scenario 2.

increases non-linearly in between $v_{c}$ and $v_{r}$, and remains to its rated generation until wind speed reaches $v_{f}$. The wind generator does not produce any power after the cut-off wind speed due to safety reasons.

The total output power for a number of wind turbines can be expressed as follows:

$$
P_{w T}=P_{w} \times N_{w}
$$

where $N_{w}$ is the number of wind generators.

\subsection{Solar generators}

Power generated by PV panels from sunlight is known as solar power. In PV panels, sunlight is converted into DC electricity. In solar power generation, the size of PV panels and solar irradiation (SI), which determines the amount of direct and defused energy on an earth surface, play a vital role. The SI, expressed as $\mathrm{kW} / \mathrm{m}^{2}$, varies from place to place and is demonstrated in Figure 4 . To obtain efficient energy transfer, the PV panels are operated with the maximum power point tracking (MPPT) mode [29].

The output power of the PV panels depends on their size and efficiency, and can be calculated as a function of SI with the assumption of operation at MPPT mode as follows:

$$
P_{s}=\eta_{s} * A * S I\left(1+\gamma\left(t_{o}-25\right)\right)
$$

where $\eta_{s}$ and $A$ are the overall efficiency and area of PV panels, respectively, $S I$ and $t_{o}$ denote solar irradiation and outside air temperature, respectively, and $\gamma$ corresponds to the temperature coefficient of the maximum output power and is generally represented as a negative percentage per ${ }^{0} C$ or $K$. The value of $\gamma$ depends on the PV technology and manufacturing parameters, it is considered as $-0.005 /{ }^{0} C$ for this study. The range of $\gamma$ for silicon cells is $0.004-0.006$ per ${ }^{0} C$ [30].

For a number of solar generators, the total output power can be extracted as follows:

$$
P_{s T}=P_{s} \times N_{s}
$$

where $N_{s}$ is number of solar generators.

\subsection{Converters}

Although converters have a range of activities, such as the ability to synchronisation with the grid, the grid current control, the MPPT control and the detection of islanding situation, in this study, converters, including 
DC/DC and DC/AC conversions, are modelled as the European weighted-efficiency $\eta_{E U R}$, given by Eq. (6), and the American weighted-efficiency $\eta_{C E C}[31,32$, given by Eq. (7), with the following equations:

$$
\begin{gathered}
\eta_{E U R}=0.03 \eta_{5 \%}+0.06 \eta_{10 \%}+0.13 \eta_{20 \%}+0.10 \eta_{30 \%}+0.48 \eta_{50 \%}+0.20 \eta_{100 \%} \\
\eta_{C E C}=0.04 \eta_{10 \%}+0.05 \eta_{20 \%}+0.12 \eta_{30 \%}+0.21 \eta_{50 \%}+0.53 \eta_{75 \%}+0.05 \eta_{100 \%}
\end{gathered}
$$

where $\eta_{w(5 \%, 10 \% \ldots o r 100 \%)}$ is the efficiency at a specified output power of the converter $P_{w, c o n}$, given as a percentage of the nominal power $P_{n}$, as follows:

$$
w(\%)=\frac{P_{w, c o n}}{P_{n}} 100 .
$$

\subsection{Energy storage systems}

Several forms of ESS, such as supercapacitors, electrochemical batteries, superconducting magnetic energy storage, compressed air energy storage and flywheel energy storage, are available in practice. These devices have different characteristics, including response times, storage capacities and peak current capabilities, which are applied for different purposes with different time-scales. Electrochemical batteries are selected in this study due to their popularity of storing electrical energy for a long time. The storage system can minimise the effect of the stochastic nature of RESs on microgrid to balance power generation and demand. This can be achieved by charging and discharging the storage system during extra and deficient energy from RESs, respectively. The charging and discharging models of the battery can be represented as follows:

$$
\begin{array}{ll}
B L(t)=B L(t-1)+\Delta t P_{c}(t) \eta_{c} & \text { if battery is charged } \\
B L(t)=B L(t-1)+\Delta t P_{d}(t) / \eta_{d} & \text { if battery is discharged }
\end{array}
$$

subject to the following battery constraints:

Power limits:

$$
\begin{aligned}
& P_{c, \text { max }}>P_{c}>0 \\
& P_{d, \text { max }}<P_{d}<0
\end{aligned}
$$

Battery energy level limits:

$$
B L_{\max }>B L(t)>B L_{\min }
$$

where $P_{c}(t)$ and $P_{d}(t)$ are the charging and discharging powers of the battery at time, $t$, respectively, $B L(t)$ and $\Delta t$ are the storage energy at time $t$ and the interval of time period, respectively, and $\eta_{c}$ and $\eta_{d}$ are the charging and discharging efficiency, respectively. For simplicity, $\eta_{c}$ and $\eta_{d}$ are assumed as unity.

The operating commands, $u(t)$, of the battery from the optimisation algorithm determine the charging and discharging energy amount. The positive values of $u(t)$ indicate $P_{c}(t)$ while negative values refer to $P_{d}(t)$.

\subsection{Loads}

The residential load varying hourly with noise, $L(t)$, is a (piecewise) continuous function with the time step of $1 \mathrm{~h}$. The time steps could be small in minute ranges, but for the sake of consistency with other literature [33, 34, 35, the time slot is taken as $1 \mathrm{~h}$. The indicative load profile for a house, which is taken from the usage 


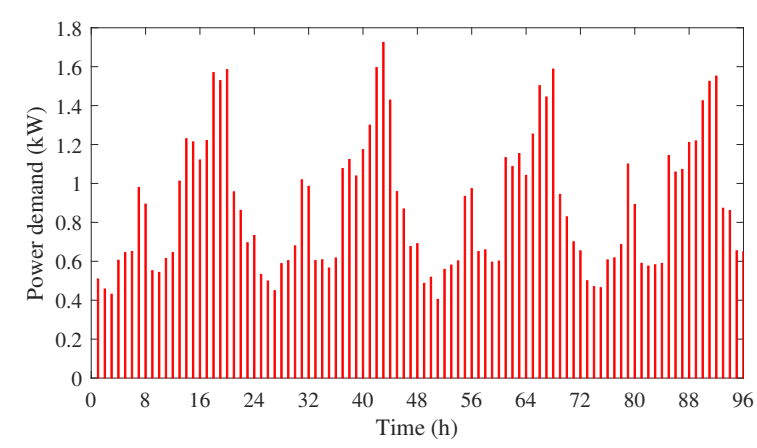

(a)

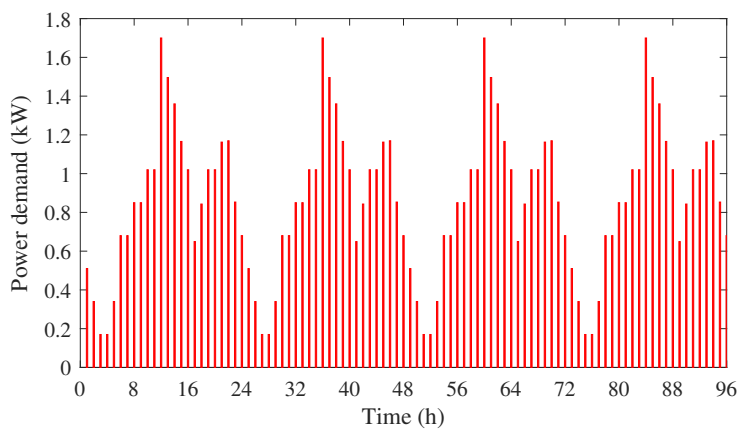

(b)

Figure 5: Indicative power demand of a householder over a time horizon of 96 hours (a) Scenario 1 and (b) Scenario 2.

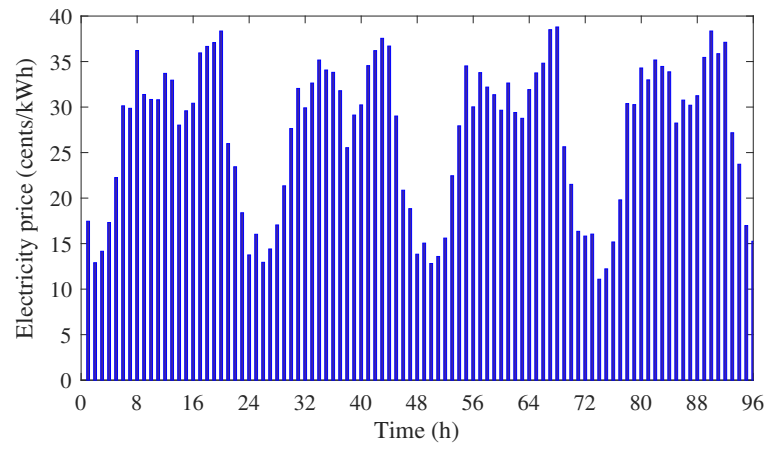

Figure 6: Real-time electricity pricing in a 96 h horizon.

of daily household appliances, is illustrated in Figure 5. The maximum load is considered around $1.8 \mathrm{~kW}$, which covers the basic loads of a house, such as refrigerators, lights, TVs, fans, computers and air-conditioning. It is assumed that the total houses are twenty, with the similar load patterns. It is observed that every day there is physical-activity routines of the householders reflected as a spike during the day time and the highest activities are carried out during the night-time, revealing cooking and relaxing activities before sleeping in Scenario 1. However, in Scenario 2, the load pattern is slightly altered to increase versatility in the simulation results.

\subsection{Electric grid} buying and selling electricity prices, which is determined in real-time for shifting loads from peak hours to off-peak hours to efficiently manage power demand, at time $t$ is identical, denoted as $C(t)(\operatorname{cents} / \mathrm{kWh})$. In real-time pricing, the rate varies over the time periods depending on wholesale market prices that are changed with respect to power demand, i.e., peak demand indicates a high rate of electricity usages 22. An indicative real-time electricity rate is demonstrated in Figure 6. The unit of the rate is expressed as cents of an Australian dollar per kilowatt hour. The rates are set to be suitably matched with the future Australian electricity market, although the variation is taken from the real-time electricity prices [36.

The import and export power at time $t$ is denoted as $P_{g}(t) \mathrm{kW}$, with the following interpretation:

- $P_{g}(t)>0$ if power is imported from the grid, and

- $P_{g}(t)<0$ if power is exported to the grid.

To reliably operate the grid network, it is assumed that power can be exchanged within the following limits. 


$$
P_{e x, \max }<P_{g}<P_{i m, \max }
$$

where $P_{e x, \max }$ and $P_{i m, \max }$ are the maximum power export (negative value) and import (positive value) limits to the grid utility, respectively. In this study, the limits are considered as $\pm 30 \mathrm{~kW}$ that a microgrid can import and export in order to maintain reliability of power supply and reduce operational costs.

\section{The proposed cost function}

The target of this study is to reduce electricity costs and make profit by exchanging the power of microgrids with the grid utility. In this case, the performance index is first formulated then the PSO algorithm is applied for solving the index in order to obtain optimal control operations of an energy storage system.

The minimum values of the performance index indicate the lowest energy costs and optimal control policies. The performance index, which is also known as objective or cost function, is generally formulated as buying and selling electricity costs of a microgrid as follows:

$$
\operatorname{Cost}_{\text {org }}(t)=P_{g} C(t)
$$
buying or selling power, is a function of battery commands, renewable energy and power demand. The $P_{g}$ as a decision variable of the optimisation algorithm can be computed from two approaches of which one is to select random grid power within given limits while another is to take random battery commands. The later is considered in this paper to optimally control the battery power.

The cost function of Eq. (10) implemented in [22, 37, 38, 23] indicates the discharging of battery power as it can guarantee the low electricity prices. Therefore, a charging term, which is the absence of the battery energy, is added in [39, 40, 33. to charge the battery from renewable energy to obtain optimal control actions of the battery as follows:

$$
\operatorname{Cost}_{\text {alt }}(t)=\sqrt{\left(\left(L(t)-P_{s T}(t)-P_{w T}(t)+u(t)\right) C(t) / C_{\min }\right)^{2}+\left(B L_{\max }-(B L+u(t))\right)^{2}}
$$

where $L(t), P_{s T}(t)$ and $P_{w T}(t)$ denote total load, total solar power and total wind power at time $t$, respectively, and $C_{\min }$ and $u(t)$ refer to the lowest electricity price and charging/discharging commands of battery energy, respectively. The first term of Eq. (11) indicates a discharging component of the battery energy, which is also related to purchasing electricity from the grid, while the second term is a charging parameter. The charging component is a critical portion for the optimal controls of the battery energy. This is because of its involvement in charging the battery during low electricity prices and the full battery charging during an upcoming event. As a result, a dynamic penalty function is proposed for the first time in this paper to complete the task in an optimal way as follows:

$$
\operatorname{Cost}_{\text {pro }}(t)=\sqrt{\left(\left(L(t)-P_{s T}(t)-P_{w T}(t)+u(t)\right) C(t)\right)^{2}+\left(f_{p c}(C)\left(B L_{\max }-(B L+u(t))\right)\right)^{2}}
$$

where $f_{p c}(C)=k-C(t)$ refers to a penalty function for charging the battery energy efficiently by determining the $k$ value, which is generally calculated as an average value of electricity prices over a day. Changing the value of $k$ has a direct effect in minimising electricity costs. The minimum values of the Cost $_{\text {pro }}$ indicate discharging the battery during low power generation and/or higher electricity prices, or charging the battery when power 
generation is high and/or prices are low. The command signals, $u$, must satisfy the battery constraints to protect immature degradation of the battery capacity, otherwise, the command is invalid and must be discarded with a high penalty cost during the optimisation process. As the cost function formulated is calculated with a time step of 1 hour (step-by-step), the algorithm evaluates the function as an on-line approach. The square root term is used to obtain a non-negative value of the cost function.

\subsection{Constraints:}

To determine the feasible solutions of the cost functions, the following constraints, equalities and inequalities, are imposed as follows.

Energy balance: The cost function must satisfy the energy balance equation as follows:

$$
P_{g}(t)+P_{s T}(t)+P_{w T}(t)=L(t)+u(t)
$$

where $u$ and $L$ are the charging/discharging power of the battery commands and load power consumption, respectively, and $P_{w T}, P_{s T}$ and $P_{g}$ are the wind, solar, and grid power, respectively. The reason of $u$ at the right-hand side of the equation is that the negative values of it indicate the discharging of the power while positive values the charging.

Battery energy: In the cost function, only $u(t)$ is varied to find the optimal solution. The commanded signal, $u(t)$, controls the battery energy as an on-line approach since the cost function proceeds step by step over the time horizon without the prior knowledge of the energy status. The values of $u(t)$ must satisfy the following constraints in order to extend the lifetime and efficiency of a battery.

1) The charging and discharging rate must be within the given limitations, i.e., $\left(P_{c, \max }<u(t)<P_{d, \max }\right)$. These limitations are imposed to decrease overheating while charging and discharging the batteries.

2) The energy level of the battery must maintain upper and lower limits, i.e., $B L_{\min }<B L+u(t)<B L_{\max }$. The lifetime of the battery depends on the status of energy levels, for example, if the battery is operated at a lower level charge or it is overcharged, the lifetime of the battery decreases.

Energy exchange: Power from the microgrid can be exchanged with the grid utility as $\pm 30 \mathrm{~kW}$.

Remarks: As there is a single decision variable, $u$, in Eq. (12), one may think to solve the problem in an accurate way through mathematical calculation in each hour by applying Lagrange multiplier, and therefore, optimisation algorithms are not needed. The reasons for the application of the optimisation algorithm are explained below.

By applying Lagrange multipliers, the objective function at a specific time can be formulated as follows:

$$
\mathrm{L}\left(u, \lambda_{1}, \lambda_{2}\right)=\sqrt{\left(\left(L-P_{s T}-P_{w T}+u\right) C\right)^{2}+\left(f_{p c}\left(B L_{\max }-(B L+u)\right)\right)^{2}}+\lambda_{1}\left(u-P_{c, \max }\right)+\lambda_{2}\left(P_{d, \min }-u\right) .
$$

The energy balance equation is not considered as equality constraint in Eq. (14) because decision variable, $u$, does not depend on the parameters.

The constraints $\left(u-P_{c, \max }\right)$ and $\left(P_{d, \min }-u\right)$ in Eq. 14 are satisfied when they are negative-valued. The 
derivatives of Eq. 14 with respect to $u, \lambda_{1}$, and $\lambda_{2}$ can be represented as follows:

$$
\begin{aligned}
\frac{\left(L-P_{s T}-P_{w T}+u\right) C-f_{p c}\left(B L_{\max }-(B L+u)\right)}{\sqrt{\left(\left(L-P_{s T}-P_{w T}+u\right) C\right)^{2}+\left(f_{p c}\left(B L_{\max }-(B L+u)\right)\right)^{2}}}+\lambda_{1}-\lambda_{2} & =0 \\
u-P_{c, \text { max }} & =0 \\
P_{d, \text { max }}-u & =0 .
\end{aligned}
$$

From Eqs. (16) and (17), it can be clearly seen that the equations can not be satisfied due to $P_{c, \max } \neq P_{d, \max }$, and consequently the $u$ can not be equal for both $P_{c, \max }$ and $P_{d, \max }$ [1]. As a result, optimisation algorithms are needed to solve the proposed problem.

\section{The PSO algorithm} toward the optimal solution.

\subsection{Standard PSO}

The most important aspect of the PSO is its straightforward calculation method that considers only two model equations of the position and velocity vectors in $N$-dimensional solution space. The movement and position of each particle $i$ can be expressed as $v_{i}^{k+1}$ vector and $x_{i}^{k+1}$ vector, respectively, as follows:

$$
\begin{aligned}
& v_{i}^{k+1}=w v_{i}^{k}+c_{1} r_{1}\left(p_{i}^{k}-x_{i}^{k}\right)+c_{2} r_{2}\left(p_{g}^{k}-x_{i}^{k}\right) \\
& x_{i}^{k+1}=x_{i}^{k}+v_{i}^{k+1}
\end{aligned}
$$

where $v_{i}^{k}$ refers to the $i^{\text {th }}$ particle velocity for $k^{\text {th }}$ iteration in $N$-dimension, $x_{i}^{k}$ denotes $i^{\text {th }}$ particle position for $k^{\text {th }}$ iteration in $N$-dimension, $p_{i}$ is the best position of an individual particle $i$ in $N$-dimension, and $p_{g}$ the best position achieved among all particles in $N$-dimension. In addition, $w$ refers to inertia weight; $r_{1}, r_{2}$ are the random numbers of the uniform distribution within the range of [0 1]; and $c_{1}, c_{2}$ represent learning factors used to control the significance of the best solution. The implementation steps of the PSO are illustrated in Figure 7. It is clear that it has a minimum number of parameters that need to be determined. One of the parameters is population size, which is often set in the range of 20-50, and the values of $w$ and $c_{1}, c_{2}$ are considered as 


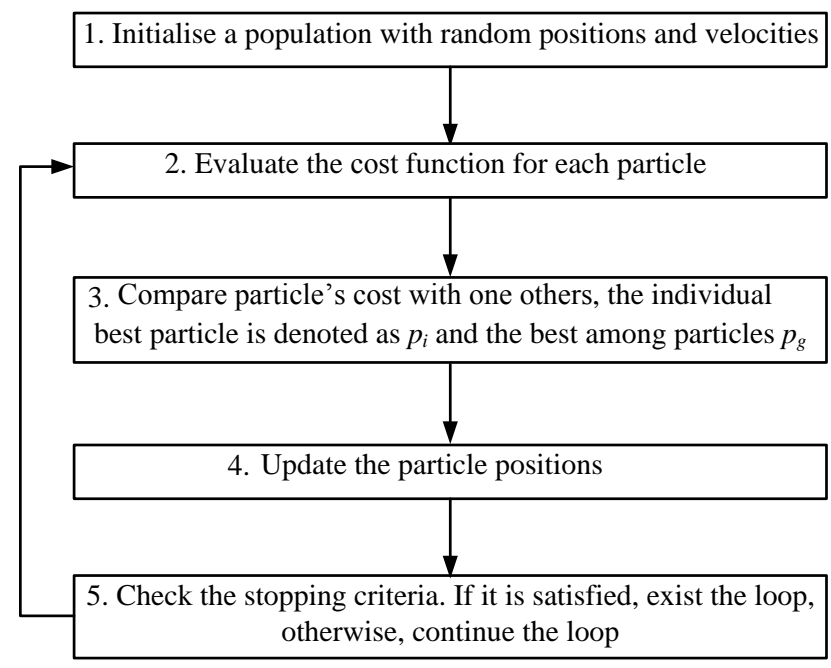

Figure 7: The standard PSO flowchart.

\subsection{Regularised $P S O$}

If particle velocities are not restricted when PSO is run, then the velocities can increase to unacceptable levels within a few iterations. Therefore, this approach was modified by the introduction of constriction coefficients to regulate particle velocities [44]. The coefficient controls the particle movements and directs them toward the convergence. The modified velocities of particles can be represented as follows:

$$
v_{i}^{k+1}=w_{C F}^{k} v_{i}^{k}+C_{1} r_{1}\left(p_{i}^{k}-x_{i}^{k}\right)+C_{2} r_{2}\left(p_{g}^{k}-x_{i}^{k}\right)
$$

where $w_{C F}=w C F$, and $C_{1}, C_{2}$ refer to cognitive and social components that have an influence on convergence speed and the finding of an optimal point in the search space. The $C_{1}$ and $C_{2}$ can be represented as follows:

$$
\begin{aligned}
& C_{1}=C F \phi_{1} \\
& C_{2}=C F \phi_{2}
\end{aligned}
$$

where

$$
\begin{array}{r}
C F=\frac{2}{\left|\phi-2+\sqrt{\phi^{2}-4 \phi}\right|} \\
\phi=\phi_{1}+\phi_{2} \\
\phi_{1}+\phi_{2} \geq 4
\end{array}
$$

where $C F$ and $\phi$ represent a constriction factor and co-efficient, respectively. Generally, $\phi$ is set 4.1 where $\phi_{1}=\phi_{2}$. Now, the constriction particles can help to converge the process toward optimal solutions without using velocity limits. However, a better approach is to use limits of velocities and positions.

\subsubsection{The procedure for the application of the PSO algorithm}

The following procedure is applied while evaluating the cost function of each hour.

Part I: Initialisation

1. Load parameters of the components according to Table 1 


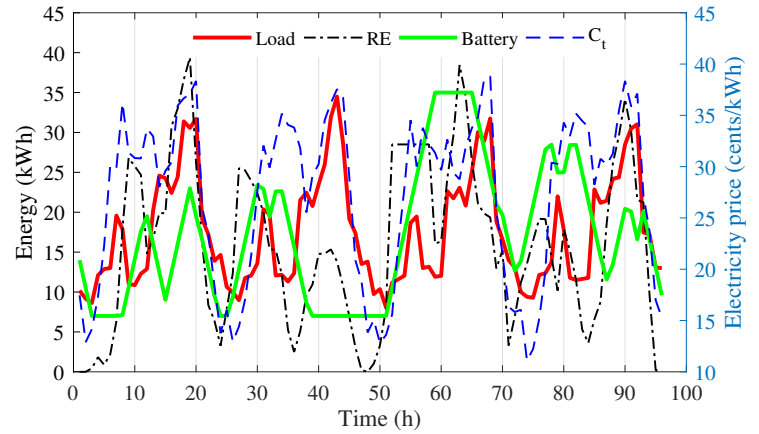

(a)

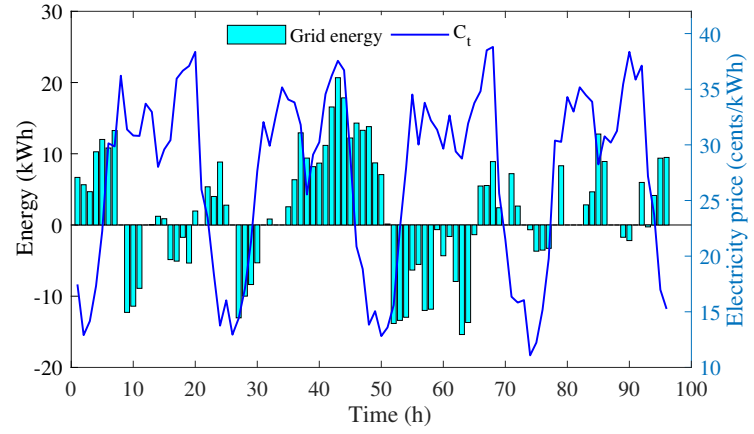

(b)

Figure 8: Original cost function for Scenario 1: (a) charging/discharging cycles of the battery energy and (b) energy exchanges with the grid.

2. Load meteorological data (hourly wind speed, solar irradiation and load profile);

3. Calculate total loads, wind power and solar power;

4. Set the parameter of the PSO:

(a) Search space dimension $=1$;

(b) Population size $=50$;

(c) Maximum number of iteration $=100$;

(d) Constriction co-efficient, $\phi=4.1$;

(e) Damping ratio of inertia coefficient, $w_{\text {damp }}=0.99$;

(f) Inertia coefficient, $w=0.73$;

(g) Penalty factor $=10^{6}$;

Part II: Iteration for the real-time evaluation

1. For each particle, the position and velocity vectors are randomly selected;

2. Evaluate the objective functions to find the fitness value of each particle;

3. Internal iteration start:

(a) Update the velocity and position of each particle according to Eqs. 20 and $\sqrt{19}$, respectively, while taking into account their limits;

(b) Calculate the objective function while all constraints are satisfied, otherwise impose penalty factor to discard the solution;

(c) Set the individual best and global best value by comparing the cost function values;

(d) Update inertia weight;

(e) Repeat steps from 3 until the termination criterion, i.e. maximum number of iterations, is satisfied. 


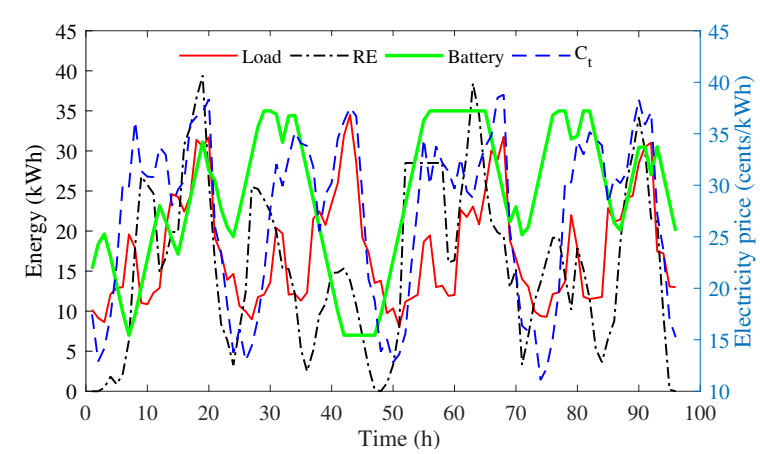

(a)

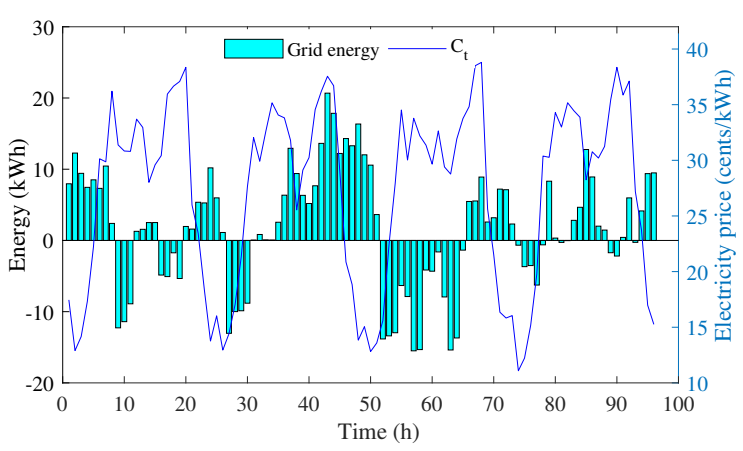

(b)

Figure 9: Alternative cost function for Scenario 1: (a) charging/discharging cycles of the battery energy and (b) energy exchange with the grid.

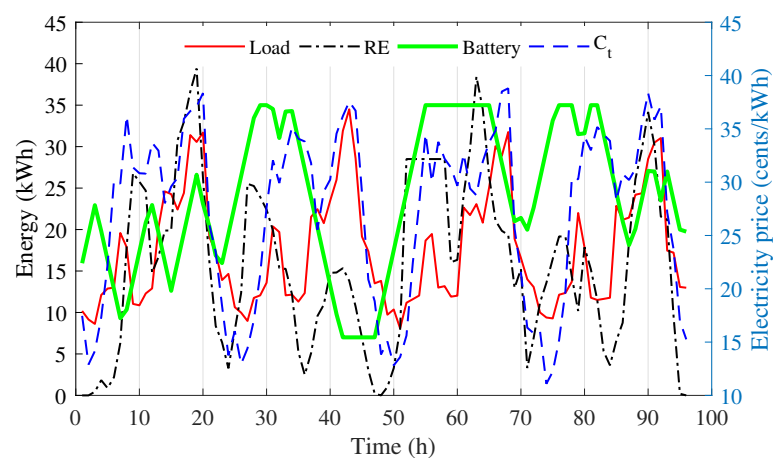

Figure 10: Proposed cost function for Scenario 1: optimal charging/discharging cycles of the battery energy. equipped with a square root, then it can continue as charging and discharging cycles as shown in Figure 8 a due 


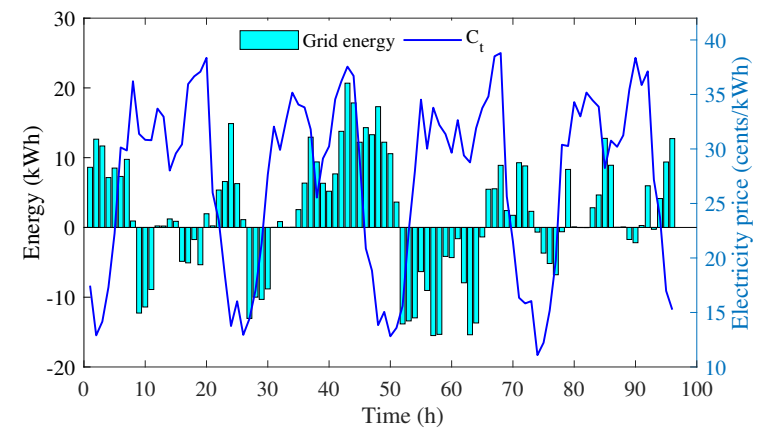

(a)

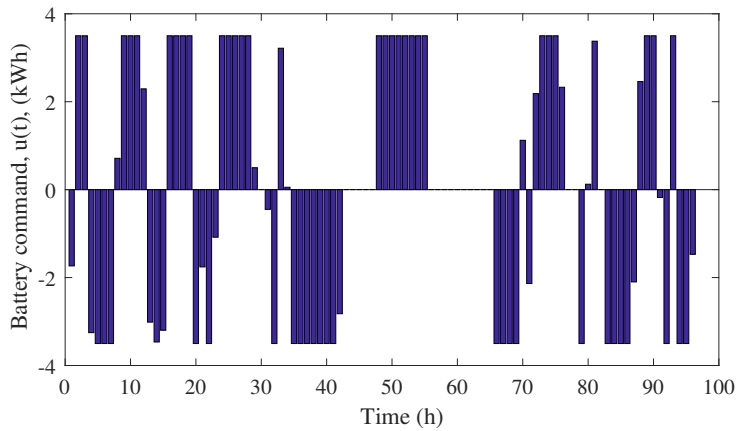

(b)

Figure 11: Proposed cost function for Scenario 1: (a) energy exchange with the grid and (b) command signals for the battery energy.

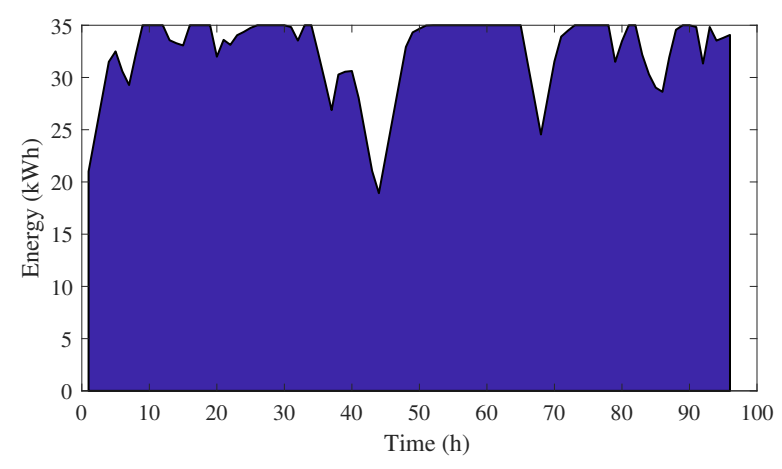

Figure 12: Reserved battery energy for special events.

to only positive values of the function. It is observed that the battery energy is being discharged from the initial time, $t=0$, to feed the loads as RESs are not producing power. The battery is only charged when renewable power is higher than the load demand, and it is discharged when load demand is higher than the local power supply. The reason for this scenario is that the cost function is developed for discharging the battery power in order to minimise electricity costs; however, the battery is charged when renewable energy is more than the load demand due to the square root terms. In this simulation, the total electricity cost for the application of renewable energy and battery is $\$ 38.59$ for a time horizon of 96 hours after actively engaging in the electricity business. It is noticeable from Figures $8 \mathrm{a}$ and $8 \mathrm{~b}$ that the algorithm does not take into account the electricity prices to charge and discharge the battery energy.

\section{Case 2: With alternative cost function}

To charge the battery from the RESs and grid utility supply, a charging term that corresponds to the absence of full charge needs to be added to incur an additional cost in the objective function. This is done mathematically as $B L_{\max }-(B L+u)$, which always provides an extra cost if the battery is not fully charged. The square root for both terms, charging and discharging, is applied at the function to charge the battery from the RESs and grid utility [39, 33, 40]. After adding the term to the objective function with a square root, the simulation results are observed in Figure 9. The battery is charged during low electricity prices up to a certain time, $t=$ $4 \mathrm{~h}$, while power is drawn from the grid utility for loads as well. After that, the battery discharges the power to feed loads to reduce electricity costs when electricity prices are high. It is also observed that the battery is charged during higher renewable power than load demand. In the alternative objective function, the algorithm 


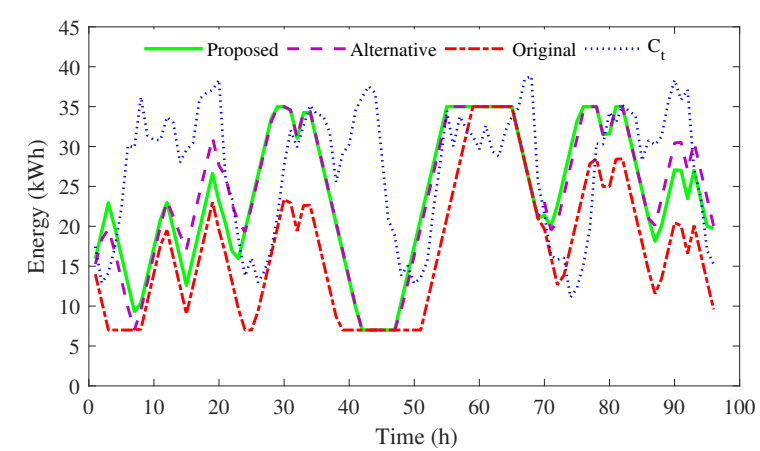

(a)

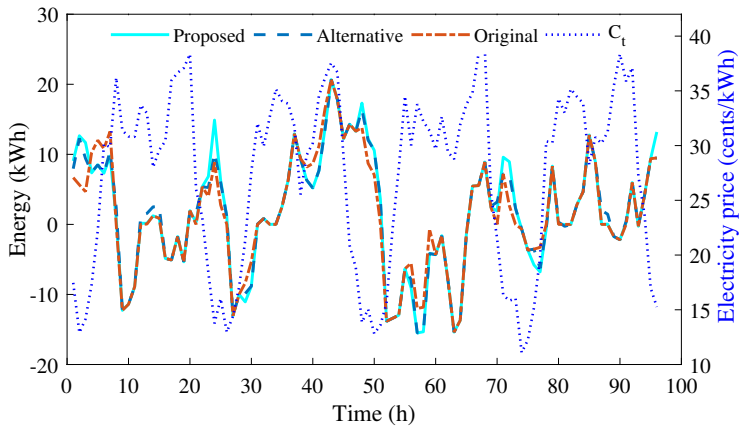

(b)

Figure 13: Comparison for Scenario 1: (a) optimal charging/discharging cycles of the battery energy and (b) energy exchange with the grid.

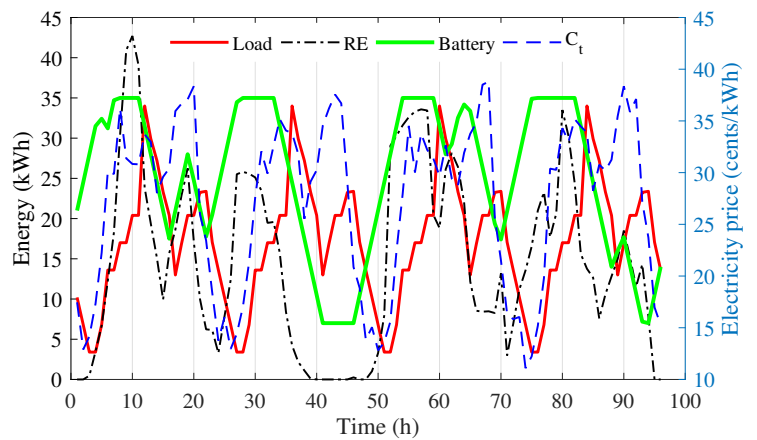

(a)

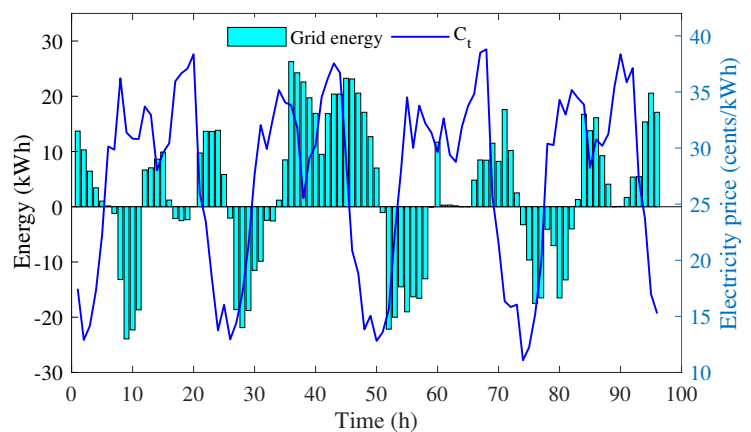

(b)

Figure 14: Proposed cost function for Scenario 2: (a) optimal charging/discharging cycles of the battery energy and (b) energy exchange with the grid.

endeavours to provide an optimal solution based on the defined cost function. However, due to the lack of a proper formulation, it still struggles to find an optimal solution. The electricity cost of this method is $\$ 36.11$ for a time horizon of 96 hours.

\section{Case 3: With proposed cost function}

The limitations of the alternative cost function can be overcome by multiplying a penalty function with the charging component for not charging the battery during low electricity prices and renewable energy availability. This penalty function can be static or dynamic based on the electricity prices. However, the static penalty function generally fails to optimally charge the battery if a varying electricity price is applied because of not following up its variation. Therefore, this paper proposes a dynamic penalty function that is based on the electricity prices as $f_{p c}(C)=k-C(t)$. The value of $k$ can be tuned for receiving the optimal operation of the battery energy. For example, if owners want to fully charge the battery, the value of $k$ needs to be increased. Generally, it is set at the average values of electricity prices, in this paper it is chosen as 29.2. The proposed penalty function is basically the opposite value of the cost function, which dynamically penalises the objective function if the battery is not charged optimally from RESs and the grid utility. The result from this cost function is demonstrated in Figures 10 and 11. It is observed that the charging and discharging cycles of the battery are similar to the alternative function, but with reduced operational costs as $\$ 33.76$ for a time horizon of 96 hours. It can be seen from Figure 10 that the storage system discharges its energy to the lowest energy level at time $t=42 \mathrm{~h}$, and after that the battery has no activities up to time $t=47 \mathrm{~h}$ due to lack of renewable 


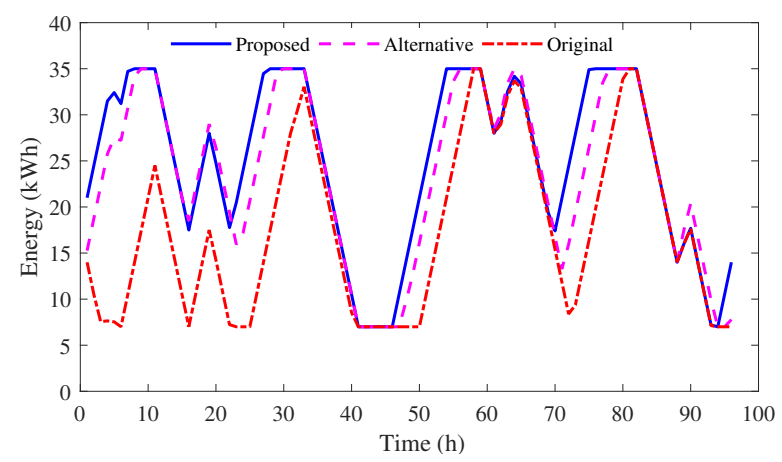

Figure 15: Comparison among proposed, alternative and original cost functions for Scenario 2.

Table 2: Comparison among cost functions in Scenario 1.

\begin{tabular}{|c|c|c|}
\hline Cost function & Electricity cost & $\%$ Saving \\
\hline Original 10 & 38.57 & 0 \\
\hline Alternative 11 & 36.11 & 6.38 \\
\hline Proposed 12 & 33.76 & 12.47 \\
\hline
\end{tabular}

power and high cost involvement in charging the battery. Similarly, the battery reaches the highest energy level at time $t=55 \mathrm{~h}$, and the battery is in an inactive mode until $t=66 \mathrm{~h}$ due to higher power generation from RESs. It should be pointed out that the formulation takes into account using the battery energy to send power to loads when power generation is less than power demand rather than selling power to the grid utility during high electricity prices. The most important part of this dynamic penalty function is that it can modify the charging and discharging cycles according to the needs of customers. For example, the battery can be fully charged by adjusting the penalty function prior to any forthcoming events, such as cloudy and rainy days, where there is a possibility of the loss of electricity connection. Taking into consideration such an event, Figure 12 depicts a full charging battery scenario for a predicted event, where the battery always tries to maintain full charge, with the expense of electricity cost $\$ 38.33$, which is still less than the original cost but with maintaining high energy levels during all the time periods. A comparative study of different functions for energy exchange with the grid utility and optimal battery control is shown in Figure 13 from which it can be seen that the charging/discharging cycles for the original cost function are different from the proposed and alternative ones.

\subsection{Scenario 2}

Although the wind speed is the same as in Figure 2, the solar irradiation and daily load patterns are changed as in Figures $4 \mathrm{~b}$ and $5 \mathrm{~b}$, respectively, to exhibit the effectiveness of the proposed function. The new solar irradiation generates less power than the previous one in Figure 4a From Figure 14 it is observed that the battery is charged during low electricity prices and high power generation, and it is discharged during low power generation and high prices. A comparison of the original, alternative and proposed functions in Scenario 2 is demonstrated in Figure 15 from where the electricity costs are tabulated in Table 3 . As can be seen from Table 3, the electricity cost for the application of the proposed cost function is $\$ 60.82$ over a time horizon of 96 hours, while other functions have higher costs than that of the proposed one. It is worth mentioning that 
Table 3: Comparison among cost functions in Scenario 2.

\begin{tabular}{|c|c|c|}
\hline Cost function & Electricity cost & $\%$ Saving \\
\hline Original 10 & 68.68 & 0 \\
\hline Alternative 11 & 62.61 & 8.84 \\
\hline Proposed (12) & 60.82 & 11.44 \\
\hline
\end{tabular}

the improvement with respect to the alternative cost function is reduced (from $6 \%$ to $3 \%$ approximately) in Scenario 2 than that of Scenario 1. This is because of variation in power generation over the peak prices. It can be concluded that the proposed cost function is superior to the other functions.

\section{Conclusion}

This paper proposes a penalty function multiplied with the charging component of the objective function in order to obtain optimal use of battery energy by controlling charging/discharging behaviours. In the proposed function, the constant value can be tuned according to the requirements of the microgrid's investors. A metaheuristic algorithm is applied to facilitate the analysis of the cost functions reported in the literature and the one proposed here. It is observed that the proposed cost function reduces electricity costs of the community microgrid by around $12 \%$ when compared with the original cost function over a time horizon of 96 hours. Numerical results and comparisons are carried out to demonstrate the effectiveness of the proposed function, and it can be concluded that the performance of the proposed cost function is superior to other functions.

In future work, the effectiveness of the proposed cost function will be evaluated in an experimental set up, where the degradation cost of a battery will be taken into account. In addition, a mathematical relationship will be established to accurately determine the $k$ value for different energy level stages.

\section{Acknowledgment}

This work is supported by the Australian Government Research Training Program (RTP) Scholarship at The University of New South Wales - Canberra, Australia.

\section{References}

[1] M. A. Hossain, H. R. Pota, M. J. Hossain, A. M. O. Haruni, Active power management in a low-voltage islanded microgrid, International Journal of Electrical Power \& Energy Systems 98 (2018) 36-47.

[2] A. S. Hassan, L. Cipcigan, N. Jenkins, Optimal battery storage operation for pv systems with tariff incentives, Applied Energy 203 (2017) 422-441.

[3] M. A. Hossain, H. R. Pota, A. M. O. Haruni, M. J. Hossain, Dc-link voltage regulation of inverters to enhance microgrid stability during network contingencies, Electric Power Systems Research 147 (2017) $233-244$. 
[4] R. H. Lasseter, Microgrids, in: Power Engineering Society Winter Meeting, 2002. IEEE, Vol. 1, IEEE, 2002, pp. 305-308.

[12] F. De Angelis, M. Boaro, D. Fuselli, S. Squartini, F. Piazza, Q. Wei, Optimal home energy management under dynamic electrical and thermal constraints, IEEE Transactions on Industrial Informatics 9 (3) (2013) $1518-1527$.

[13] A. C. Luna, N. L. Diaz, F. Andrade, M. Graells, J. M. Guerrero, J. C. Vasquez, Economic power dispatch of distributed generators in a grid-connected microgrid, in: Power Electronics and ECCE Asia (ICPE-ECCE Asia), 2015 9th International Conference on, IEEE, 2015, pp. 1161-1168.

[14] A. C. L. Hernández, N. L. D. Aldana, M. Graells, J. C. V. Quintero, J. M. Guerrero, Mixed-integer-linearprogramming-based energy management system for hybrid pv-wind-battery microgrids: Modeling, design, and experimental verification, IEEE Transactions on Power Electronics 32 (4) (2017) 2769-2783.

[15] Y. Choi, H. Kim, Optimal scheduling of energy storage system for self-sustainable base station operation considering battery wear-out cost, Energies 9 (6) (2016) 462.

[16] A. Chaouachi, R. M. Kamel, R. Andoulsi, K. Nagasaka, Multiobjective intelligent energy management for a microgrid, IEEE Transactions on Industrial Electronics 60 (4) (2013) 1688-1699.

[17] Q. Wei, G. Shi, R. Song, Y. Liu, Adaptive dynamic programming-based optimal control scheme for energy storage systems with solar renewable energy, IEEE Transactions on Industrial Electronics 64 (7) (2017) $5468-5478$. 
[18] S. Teleke, M. E. Baran, S. Bhattacharya, A. Q. Huang, Rule-based control of battery energy storage for dispatching intermittent renewable sources, IEEE Transactions on Sustainable Energy 1 (3) (2010) $117-124$.

[19] Y. Riffonneau, S. Bacha, F. Barruel, S. Ploix, Optimal power flow management for grid connected pv systems with batteries, IEEE Transactions on Sustainable Energy 2 (3) (2011) 309-320.

[20] O. Penangsang, A. Soeprijanto, A. A. Fitriana, E. S. Ningrum, Operation optimization stand-alone microgrid using firefly algorithm considering lifetime characteristics of battery, in: Intelligent Technology and Its Applications (ISITIA), 2016 International Seminar on, IEEE, 2016, pp. 565-570.

${ }_{400}[21]$ B. V. Mbuwir, F. Ruelens, F. Spiessens, G. Deconinck, Battery energy management in a microgrid using batch reinforcement learning, Energies 10 (11) (2017) 1846.

[22] T. Huang, D. Liu, Residential energy system control and management using adaptive dynamic programming, in: Neural Networks (IJCNN), The 2011 International Joint Conference on, IEEE, 2011, pp. 119-124.

[23] G. Shi, Q. Wei, D. Liu, Optimization of electricity consumption in office buildings based on adaptive dynamic programming, Soft Computing 21 (21) (2017) 6369-6379.

[24] H. Borhanazad, S. Mekhilef, V. G. Ganapathy, M. Modiri-Delshad, A. Mirtaheri, Optimization of microgrid system using mopso, Renewable Energy 71 (2014) 295-306.

[25] C. Justus, Wind energy statistics for large arrays of wind turbines (new england and central us regions), Solar Energy 20 (5) (1978) 379-386.

[26] S. Rehman, N. M. Al-Abbadi, Wind shear coefficients and energy yield for dhahran, saudi arabia, Renewable Energy 32 (5) (2007) 738-749.

[27] R. Farrugia, The wind shear exponent in a mediterranean island climate, Renewable Energy 28 (4) (2003) $647-653$.

[28] B. S. Borowy, Z. M. Salameh, Optimum photovoltaic array size for a hybrid wind/pv system, IEEE Transactions on energy conversion 9 (3) (1994) 482-488.

[29] W. Xiao, M. G. Lind, W. G. Dunford, A. Capel, Real-time identification of optimal operating points in photovoltaic power systems, IEEE Transactions on Industrial Electronics 53 (4) (2006) 1017-1026.

[30] A. Kaabeche, M. Belhamel, R. Ibtiouen, Sizing optimization of grid-independent hybrid photovoltaic/wind power generation system, Energy 36 (2) (2011) 1214-1222.

[31] Overall efficiency of photovoltaic inverters, European Standard EN 50530, 2010.

[32] A. C. Nanakos, E. C. Tatakis, N. P. Papanikolaou, A weighted-efficiency-oriented design methodology of flyback inverter for ac photovoltaic modules, IEEE Transactions on Power Electronics 27 (7) (2012) 3221-3233. 
[33] D. Fuselli, F. De Angelis, M. Boaro, S. Squartini, Q. Wei, D. Liu, F. Piazza, Action dependent heuristic dynamic programming for home energy resource scheduling, International Journal of Electrical Power \& Energy Systems 48 (2013) 148-160.

[34] A. Bakirtzis, P. Dokopoulos, Short term generation scheduling in a small autonomous system with unconventional energy sources, IEEE Transactions on power systems 3 (3) (1988) 1230-1236.

[35] D. Liu, Y. Xu, Q. Wei, X. Liu, Residential energy scheduling for variable weather solar energy based on adaptive dynamic programming, IEEE/CAA Journal of Automatica Sinica 5 (1) (2018) 36-46.

[36] ComEd, USA, http://www.thewattspot.com/.

[37] M. Boaro, D. Fuselli, F. De Angelis, D. Liu, Q. Wei, F. Piazza, Adaptive dynamic programming algorithm for renewable energy scheduling and battery management, Cognitive Computation 5 (2) (2013) 264-277.

[38] G. Shi, Q. Wei, D. Liu, An adaptive dynamic programming based method for optimization of electricity consumption in office buildings, in: Neural Networks (IJCNN), 2016 International Joint Conference on, IEEE, 2016, pp. 4551-4556.

[39] S. Squartini, M. Boaro, F. De Angelis, D. Fuselli, F. Piazza, Optimization algorithms for home energy resource scheduling in presence of data uncertainty, in: Intelligent Control and Information Processing (ICICIP), 2013 Fourth International Conference on, IEEE, 2013, pp. 323-328.

[40] N. Gudi, L. Wang, V. Devabhaktuni, A demand side management based simulation platform incorporating heuristic optimization for management of household appliances, International Journal of Electrical Power \& Energy Systems 43 (1) (2012) 185-193.

[41] H. P. Gavin, J. T. Scruggs, Constrained optimization using lagrange multipliers, CEE 201L. Duke University.

[42] J. Kennedy, R. Eberhart, C. 1995. particle swarm optimization, in: IEEE International Conference on Neural Networks (Perth, Australia), IEEE Service Center, Piscataway, NJ, pp. 1942-1948.

[43] R. Poli, J. Kennedy, T. Blackwell, Particle swarm optimization, Swarm intelligence 1 (1) (2007) 33-57.

[44] M. Clerc, J. Kennedy, The particle swarm-explosion, stability, and convergence in a multidimensional complex space, IEEE transactions on Evolutionary Computation 6 (1) (2002) 58-73.

${ }_{450}^{4}[45]$ T. Huang, D. Liu, A self-learning scheme for residential energy system control and management, Neural Computing and Applications 22 (2) (2013) 259-269. 